\title{
The Swansea Floating Endoscopic Assistant: A Surgical Handling Aid
}

\author{
Jayan George $^{1, * \mathbb{D}}$, Amir Farboud ${ }^{2}$, Hassan A Elhassan ${ }^{2} \mathbb{D}$ and Heikki B Whittet ${ }^{2}$ \\ 1 General Surgical Department, Sheffield Teaching Hospitals NHS Foundation Trust, Herries Road, \\ Sheffield S5 7AU, UK \\ 2 ENT Head and Neck surgery department, Singleton Hospital, Swansea SA2 8QA, UK; \\ amirfarboud@doctors.net.uk (A.F.); hassanelhassan@gmail.com (H.A.E.); \\ heikki.whittet@wales.nhs.uk (H.B.W.) \\ * Correspondence: jayan.george@aol.com; Tel.: +44-1142-434-343; Fax: +44-1142-266-986
}

Received: 11 October 2018; Accepted: 4 November 2018; Published: 7 November 2018

check for updates

\begin{abstract}
Endoscopic sinus surgery is a rapidly advancing area of Otolaryngology. Operations can be lengthy and are often performed by a single surgeon. Repetitive movements can also lead to muscular fatigue. To mitigate against this, we regularly deploy two retractable Flexi ${ }^{\mathrm{TM}}$ compact leads, one bigger than the other connected together and suspended from the theatre operating light arm using velcro ties. The leads are then wrapped around the endoscope using a cotton crepe bandage, in a double loop with a reef knot. The larger lead attaches to the focus/zoom adjustment part of the endoscopic camera, and the smaller lead attaches to the scope $20 \mathrm{~cm}$ from the tip allowing the scope to float when suspended, with a slight downward tilt towards the patient. The mechanical effect this produces allows an advantageous reduction in the scope's weight from $404 \mathrm{~g}$ un-suspended, to $65 \mathrm{~g}$ with the setup described. This subsequently reduces stress on the elbows, shoulders neck and lumbar spine. The Swansea Floating Endoscopic Assistant adheres to basic ergonomic principles and has the potential for application in other areas of Otorhinolaryngology and Skull Base Surgery.
\end{abstract}

Keywords: surgical procedures; operative; nose; surgery

\section{Introduction}

Sino-nasal surgery has undergone many advances over the last hundred years with the introduction of the endoscope [1]. Single surgeon endoscopic sinus surgery (ESS) is performed with the operator holding the endoscope in the non-dominant hand with instruments in the other hand. Constant weight bearing, handling and manipulation of the camera-endoscope assembly unit for protracted periods of time can result in operator fatigue or discomfort. It has been noted that performance can be adversely affected with increased muscular workload [2].

Systematic reviews and ergonomic analyses looking at the musculoskeletal stresses, and how they affect endoscopic nasal surgeons are lacking. There are, however, principles derived from general ergonomic knowledge that can be applied in the context of ESS. Statham et al. performed an ergonomic analysis of musculoskeletal fatigue in microsurgery for Otolaryngologists. The setup is slightly different to ESS procedures but the principles are similar. High-risk postures that can induce further musculoskeletal injury are those that add strain to the neck and torsional forces to the shoulders. Ideal postures retain the arms and feet supported with shoulders in a not-raised, neutral anatomic position [3]. The National Institute of Occupational Safety and Health identified individuals who work with static loads, involved in repetitive movements and identified that they were more likely to experience of work-related musculoskeletal disorders of the neck, upper extremity and lower back [4]. A recent survey focusing on ESS revealed $80 \%$ of surgeons experience musculoskeletal 
symptoms [5]. The health and safety executive estimate 8.9 million working days are lost due to work related disorders in the United Kingdom [6].

One ESS operation can take up to two hours and one surgeon may perform multiple operations in the course of a full day operating list [7]. This repetitive stress predisposes a surgeon to musculoskeletal injury. A recent study showed that $83 \%$ of Otolaryngologists are affected by musculoskeletal problems [8]. Musculoskeletal injury is also prevalent in laparoscopic surgery and a 2009 ergonomic study showed that $86.9 \%$ of laparoscopic surgeons experienced physical discomfort from operative surgery [9].

In order to address these ergonomic issues, our group has developed the Swansea Floating Endoscopic Assistant (SFEA) with the aim of reducing operator strain and muscular fatigue.

\section{Methods/Surgical Technique}

This technique is used with the surgeon in the standing position. We accept that ESS can be performed sitting, but in our experience, and due to our theatre setup and training, all our surgeons stand for ESS. The setup includes two retractable Flexi ${ }^{\mathrm{TM}}$ compact leads (Flexi, Charlotte, North Carolina, United States) one bigger than the other are joined together using a cotton tie (Figure 1). A velcro tie is used to attach the larger Flexi retractor to the arm of the operating theatre light, thus suspending it. This allows the two retractors to line up in a vertical plane, with the large device higher than the smaller one so they can operate in synchrony unimpeded (Figure 2). Leads are wrapped around the endoscope using cotton crepe bandage, in a double loop with reef knot (Figures 3 and 4). The larger lead attaches to the focus/zoom adjustment part of the endoscopic camera, and the smaller lead attaches to the scope $20 \mathrm{~cm}$ from the tip from the tip around the locking mechanism of the flat Stammberger telescope handle (Storz, Tuttlingen, Germany). In this way the user can switch between different angled endoscopes to view all relevant sinuses without significant interruption. The setup allows the endoscope to float when suspended with a downward tilt towards the patient. Mechanical advantage reduces the scope's weight from $404 \mathrm{~g}$ unsuspended to $65 \mathrm{~g}$ with the setup as described. This makes the equipment nearly weightless to the operator, and does not confine movement. The leads are cleaned but not sterilised between procedures.

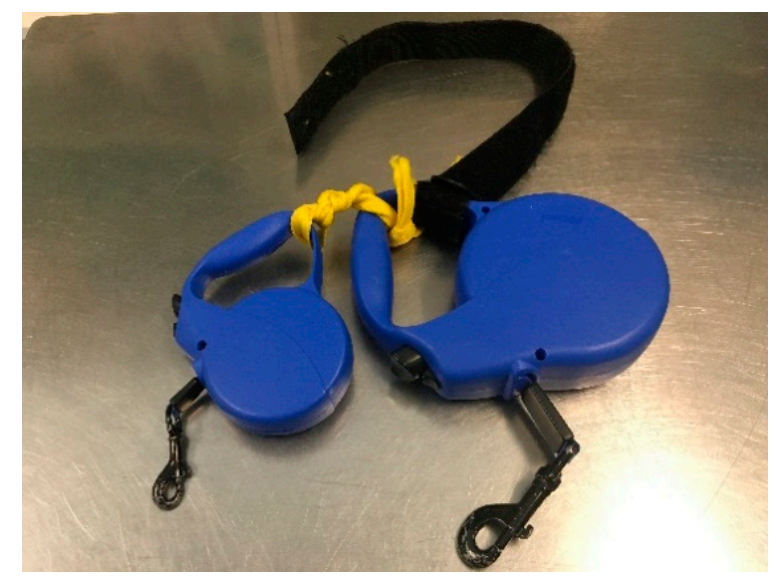

Figure 1. Two retractable Flexi (TM) compact leads attached together with velcro tie in preparation for mounting.

The device we use is unlicensed, and although we are aware of this we have not had any problems in its deployment in approximately 600 procedures over the last 10 years for endoscopic sinus procedures including complex frontal sinus explorations (Draf 2b-3, Modified Lothrop procedure) and combined approach fronto-ethmoidectomy with good effect, and in the vast majority of cases excellent outcomes. The trainees who have used this technique have found this to aid their movements and has reduced musculoskeletal stress at the end of an all-day operating list. Our department represents the 
tertiary referral centre for complex sinus surgery in South Wales, UK and so our experience is from a breadth of referrals across a wide demographic.

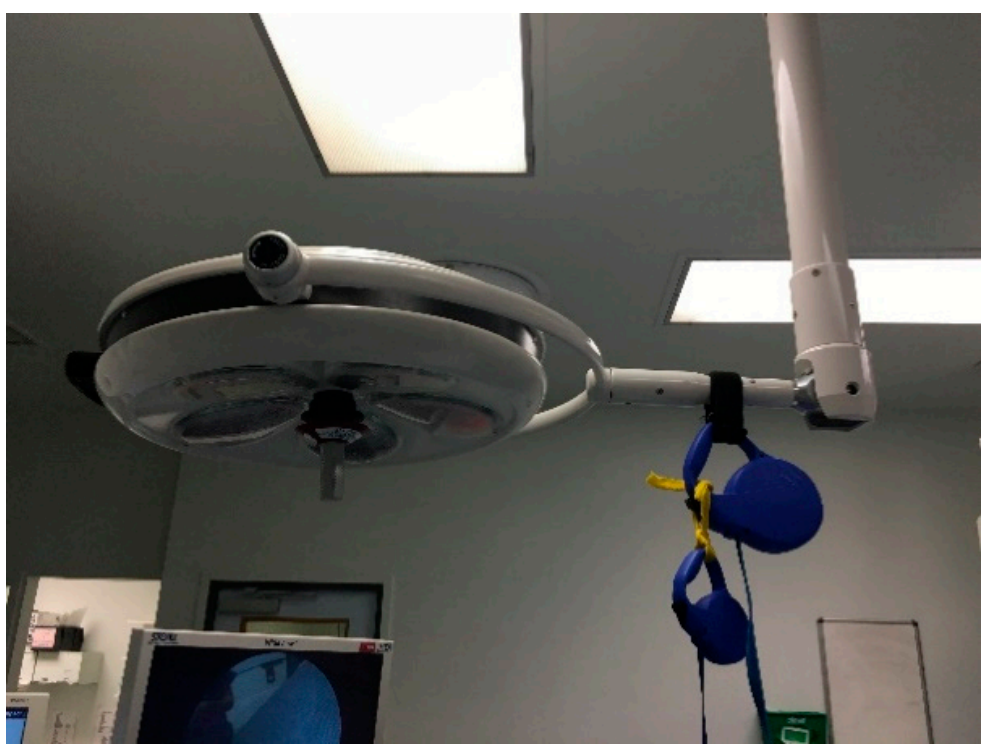

Figure 2. The retractable compact leads suspended from operating theatre light arm.

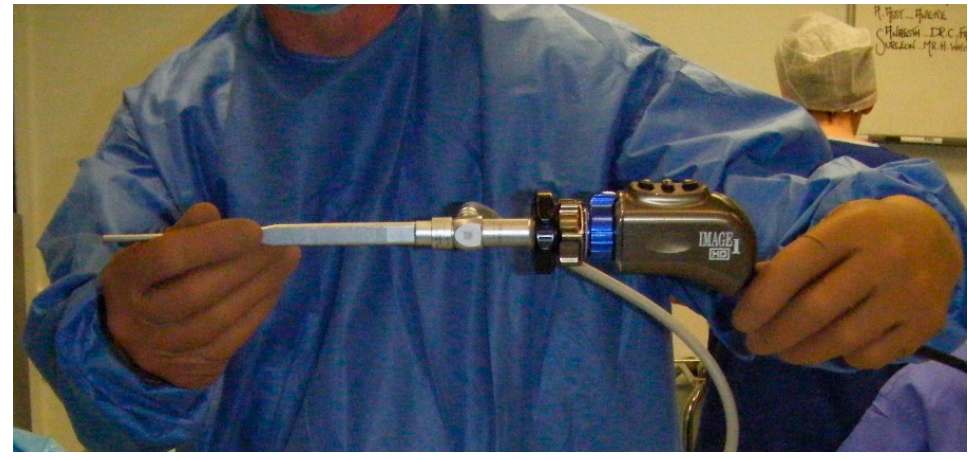

Figure 3. Endoscope camera setup prior to connection with retractable assembly.

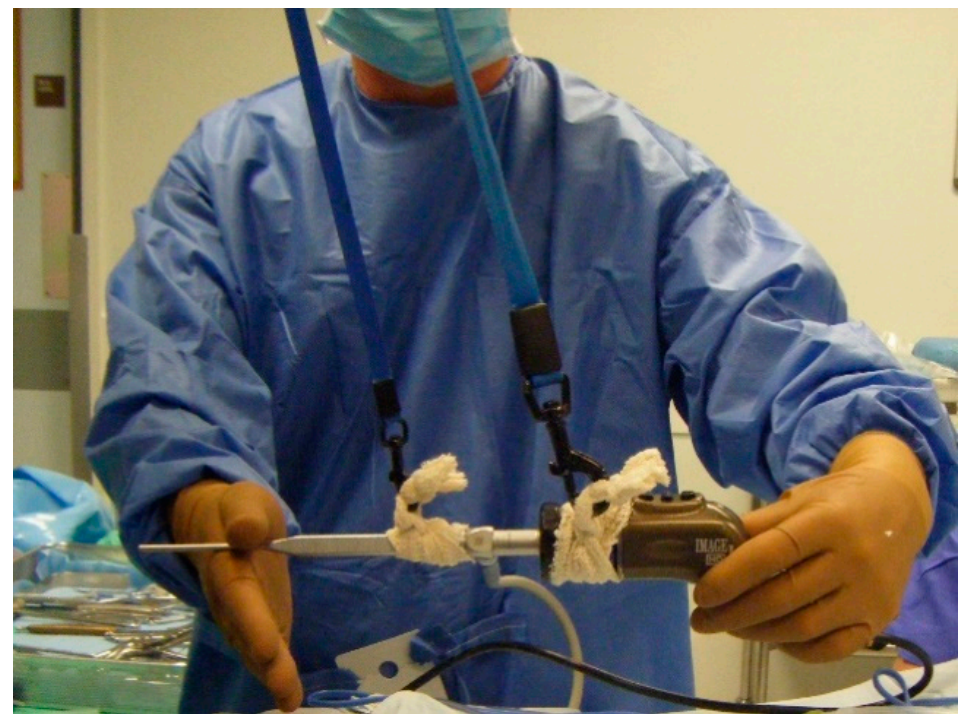

Figure 4. Endoscope camera setup connected to retractable assembly by 2 crepe bandages secured with 2 reef knots at each end. Note the larger retractable lead is attached to the camera end and the smaller lead is attached to the endoscope end. 


\section{Discussion}

There are increasing accounts of surgeons suffering from operative related physical discomfort. A 2011 survey showed that 77\% of surgeons performing ESS suffer from physical discomfort and the location of this discomfort was mainly in the shoulders, arms and neck. $63 \%$ reported lacking knowledge of ergonomic principles. The same study also highlighted the lack of information regarding sinus surgery ergonomics and the need to reduce the long-term repetitive strain injuries [10]. Another study reported of those that experiences musculoskeletal discomfort $60 \%$ were due to neck and back [5].

A systematic review of ergonomics in ESS found that an arm support could be beneficial as this minimised the stress on the neck, shoulder and lumbar spine [11]. While we accept that an arm support (such as a covered mayo table) either deployed when the operator is sitting or standing is a useful adjunct, however our technique is a better assist to ESS by reducing the effective weight of the endoscope and therefore allowing greater range of motion for the operating surgeon (Figure 5).

Lessons learned from laparoscopic surgery to ESS include adjusting the table to keep hands in line with elbow $+/-10 \mathrm{~cm}$, keeping the arms slightly abducted and internally rotated, limiting wrist flexion, deviation and rotation to $<15$ degrees and gently resting the shaft of the endoscope at superior nasal vestibule when able [12]. Our described technique is setup to keep the elbows in line with the hands, and given the nature of the straps, the natural resting position is one of slight abduction in the arms and internal rotation which limits wrist flexion while the straps take the weight. This reduces the stress on the shoulders, arms and neck (Figures 5 and 6). Through the mechanical advantage of using pulleys, this system provides an effective reduction in weight of the rigid endoscope therefore reducing muscular and neurophysiological fatigue. This is further supported by studies demonstrating an association between prolonged neck flexion and the presence of neck pain $[13,14]$. The relaxed neck and shoulder position maintains neutral posture and limits the strain on these core muscles (Figure 6).

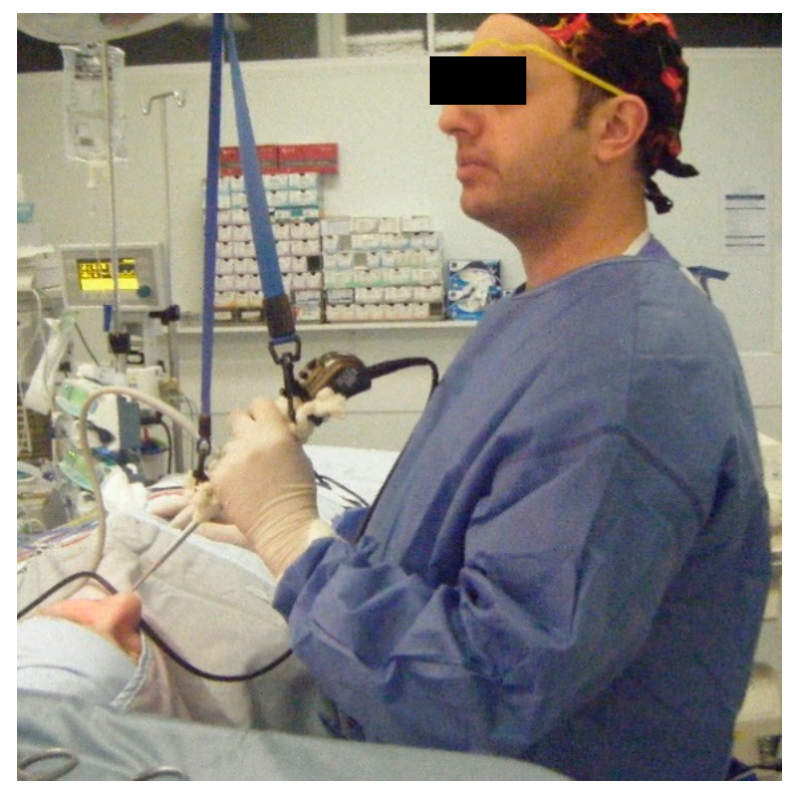

Figure 5. Shows the author utilising the SFEA with arm at 90 degrees to elbow and the wrist relaxed and able to flex and extend freely without undue tension or prolonged contraction thereby minimising fatigue. 


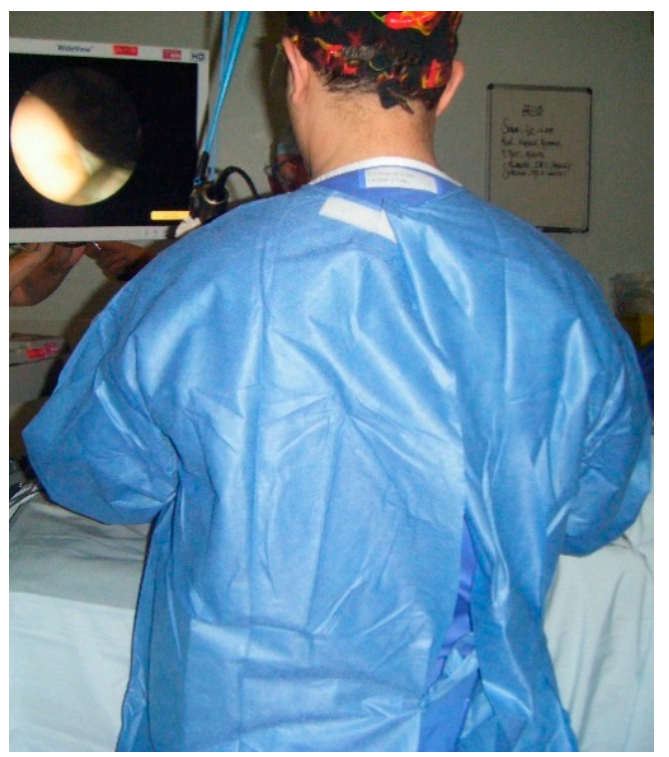

Figure 6. Shows the author deploying the SFEA and maintaining an ergonomic posture with minimal abduction of the arms.

\section{Conclusions}

The muscular strain encountered through performing ESS can be significant over the course of a career, due in part to non-adherence to ergonomic principles. The SFEA alleviates some strain and allows for the unencumbered and fluid movements needed during ESS. The SFEA has the potential for application in other areas of otolaryngology, such as Skull Base and Endoscopic Ear Surgery. The SFEA is not a finished product which needs to conform to operative standards of safety and hygiene, however its advantages highlight the need for such devices through improved ergonomics resulting in better surgeon performance.

Author Contributions: J.G.: Preparing the manuscript, analysing the data, editing and proofing. A.F.: Editing, concept development and proofing. H.A.E.: Editing, concept development and proofing. H.B.W.: Editing, concept development and proofing.

Funding: This research received no external funding.

Conflicts of Interest: The authors declare no conflict of interest.

\section{References}

1. Chandra, R.K.; Conley, D.B.; Kern, R.C. Evolution of the Endoscope and Endoscopic Sinus Surgery. Otolaryngol. Clin. N. Am. 2009, 42, 747-752. [CrossRef] [PubMed]

2. Manasnayakorn, S.; Cuschieri, A.; Hanna, G.B. Ideal manipulation angle and instrument length in hand-assisted laparoscopic surgery. Surg. Endosc. 2008, 22, 924-929. [CrossRef] [PubMed]

3. Statham, M.M.; Sukits, A.L.; Redfern, M.S.; Smith, L.J.; Sok, J.C.; Rosen, C.A. Ergonomic analysis of microlaryngoscopy. Laryngoscope 2010, 120, 297-305. [CrossRef] [PubMed]

4. Bernard, B.P. Musculoskeletal Disorders and Workplace Factors. A Critical Review of Epidemiologic evidence for Work-Related Musculoskeletal Disorders of the Neck, Upper Extremity, and Low Back. 1997. Available online: https:/ / stacks.cdc.gov/view/cdc/21745 (accessed on 6 November 2018).

5. Rimmer, J.; Amin, M.; Fokkens, W.J.; Lund, V.J. Endoscopic sinus surgery and musculoskeletal symptoms. Rhinol. J. 2016, 54, 105-110. [CrossRef] [PubMed]

6. Health and Safety Executive. Work-Related Musculoskeletal Disorders (WRMSDs) Statistics in Great Britain 2017. Available online: www.hse.gov.uk/statistics/ (accessed on 20 October 2018).

7. Piédrola Maroto, D.; Jiménez Puente, A.; Bandera Florido, A.; Perea-Milla López, E.; Conde Jiménez, M.; Pons Palliser, J.; Casado Morente, J.C.; Povedano Rodríguez, V. Clinical and performance results of functional endoscopic sinus surgery. Acta Otorrinolaringol. Esp. 2004, 55, 320-326. [CrossRef] 
8. Wong, A.; Baker, N.; Smith, L.; Rosen, C.A. Prevalence and risk factors for musculoskeletal problems associated with microlaryngeal surgery: A. national survey. Laryngoscope 2014, 124, 1854-1861. [CrossRef] [PubMed]

9. Park, A.; Lee, G.; Seagull, F.J.; Meenaghan, N.; Dexter, D. Patients Benefit While Surgeons Suffer: An Impending Epidemic. J. Am. Coll. Surg. 2010, 210, 306-313. [CrossRef] [PubMed]

10. Little, R.M.; Deal, A.M.; Zanation, A.M.; McKinney, K.; Senior, B.A.; Ebert, C.S., Jr. Occupational hazards of endoscopic surgery. Int. Forum Allergy Rhinol. 2012, 2, 212-216. [CrossRef] [PubMed]

11. Ayad, T.; Péloquin, L.; Prince, F. Ergonomics in endoscopic sinus surgery: Systematic review of the literature. J. Otolaryngol. 2005, 34, 333-340. [CrossRef] [PubMed]

12. Ramakrishnan, V.R.; Montero, P.N. Ergonomic Considerations in Endoscopic Sinus Surgery: Lessons Learned from Laparoscopic Surgeons. Am. J. Rhinol. Allergy 2013, 27, 245-250. [CrossRef] [PubMed]

13. Finsen, L.; Christensen, H.; Bakke, M. Musculoskeletal disorders among dentists and variation in dental work. Appl. Ergon. 1998, 29, 119-125. [CrossRef]

14. Szeto, G.P.Y.; Straker, L.; Raine, S. A field comparison of neck and shoulder postures in symptomatic and asymptomatic office workers. Appl. Ergon. 2002, 33, 75-84. [CrossRef]

(C) 2018 by the authors. Licensee MDPI, Basel, Switzerland. This article is an open access article distributed under the terms and conditions of the Creative Commons Attribution (CC BY) license (http:/ / creativecommons.org/licenses/by/4.0/). 